\title{
COHOMOLOGICAL DIMENSION AND APPROXIMATE LIMITS
}

\author{
LEONARD R. RUBIN
}

(Communicated by James West)

\begin{abstract}
Approximate (inverse) systems of compacta have been useful in the study of covering dimension, dim, and cohomological dimension over an abelian group $G, \operatorname{dim}_{G}$. Such systems are more general than (classical) inverse systems. They have limits and structurally have similar properties. In particular, the limit of an approximate system of compacta satisfies the important property of being an approximate resolution. We shall prove herein that if $G$ is an abelian group, a compactum $X$ is the limit of an approximate system of compacta $X_{a}, n \in \mathbb{N}$, and $\operatorname{dim}_{G} X_{a} \leq n$ for each $a$, then $\operatorname{dim}_{G} X \leq n$.
\end{abstract}

\section{INTRODUCTION}

In this paper, a compactum will always mean a compact Hausdorff space, and by map we mean a continuous function. When we use the term polyhedron, we will mean the space of some simplicial complex and which has the weak topology. One should keep in mind that every CW-complex has the homotopy type of a polyhedron, and that a polyhedron with the metric topology induced by this triangulation is homotopy equivalent to itself under the CW-topology (cf., Appendix of [MS]).

The theory of approximate (inverse) systems began in [MR1] and was used in the study of cohomological dimension with integer coefficients in [MR2]. Since the publication of those papers, much progress has been made in developing the concepts involved in such systems and their limits ([Ma]). In particular, a revised version of this theory applicable to arbitrary spaces ([MW]), and which we shall employ herein, has been quite useful. Instead of the numerical meshes required in the original definitions, the latter uses open cover meshes. It has been shown ([MRU], [Wa]) that this new approach is an extension of that of its predecessor. Hence, the results which we prove below will be valid for the type of systems defined in [MR1] whenever the hypotheses apply.

The authors proved in [MR1] that if $X$ is the limit of an approximate system of metric compacta $X_{a}, n \in \mathbb{N}$, and $\operatorname{dim} X_{a} \leq n$ for all $a$, then $\operatorname{dim} X \leq n$. The technique used there was quite special, employing the "covering" approach to dimension theory, so it cannot be applied directly for other types of dimension such as cohomological dimension. Recently ([DD]), another more general notion has been brought into focus. The idea is to consider a polyhedron $K$ and the statement that for a given space $X, K \in \operatorname{AE} X$. The latter means that for each closed subset

Received by the editors November 16, 1995.

1991 Mathematics Subject Classification. Primary 54F45, 55M10, 54B35.

Key words and phrases. Dimension, cohomological dimension, Eilenberg-Mac Lane complex, approximate (inverse) system, inverse system, resolution, approximate resolution.

(C) 1997 American Mathematical Society 
$A$ of $X$ and each map $f: A \rightarrow K$ from a closed subset $A$ of $X$, there exists a map $F: X \rightarrow K$ extending $f$. This is sometimes written $X \tau K$, which we prefer. Indeed, this way of thinking can already be found in [DR]. To put it into perspective, note that for any paracompact space $X, X \tau S^{n}$ is equivalent to $\operatorname{dim} X \leq n$. Analogously, if one replaces $S^{n}$ by an Eilenberg-Mac Lane complex $K(G, n)$ where $G$ is an abelian group ([Wh]), then one gets the statement that $X \tau K(G, n)$ if and only if $\operatorname{dim}_{G} X \leq$ $n$. Here, $\operatorname{dim}_{G}$ refers to cohomological dimension with coefficients in the abelian group $G$ (see the Appendix of [Na]).

The main result of this paper is the following.

1.1. Theorem. Let $K$ be a polyhedron, $\mathbf{X}=\left\{X_{a}, \mathcal{U}_{a}, p_{a, a^{\prime}}, A\right\}$ be an approximate system of compacta such that for each $a \in A, X_{a} \tau K$, and let $X=\operatorname{limX}$. Then $X \tau K$.

One then has as a corollary the result we wanted to establish.

1.2. Corollary. Let $\mathbf{X}=\left\{X_{a}, \mathcal{U}_{a}, p_{a, a^{\prime}}, A\right\}$ be an approximate system of compacta, $X=\lim \mathbf{X}$, and $n \in \mathbb{N}$. If for each $a \in A$, $\operatorname{dim} X_{a} \leq n$, then $\operatorname{dim} X \leq n$. If $G$ is an abelian group and for each $a \in A, \operatorname{dim}_{G} X_{a} \leq n$, then $\operatorname{dim}_{G} X \leq n$.

\section{Proof of the Main Result}

For convenience, we restate the requirements $[\mathrm{MW}]$ for $\mathbf{X}=\left\{X_{a}, \mathcal{U}_{a}, p_{a, a^{\prime}}, A\right\}$ to be an approximate system with open cover meshes $\mathcal{U}_{a}$. First, one must have a directed set $A=(A, \leq)$ which is unbounded and so that for each $a \in A, X_{a}$ is a space with a normal open cover $\mathcal{U}_{a}$ of $X_{a}$. Whenever $a \leq a^{\prime}$, we require a unique map $p_{a, a^{\prime}}: X_{a^{\prime}} \rightarrow X_{a}$ subject to:

(A1) $d\left(p_{a, a^{\prime}} p_{a^{\prime}, a^{\prime \prime}}, p_{a, a^{\prime \prime}}\right) \leq \mathcal{U}_{a}$ for $a<a^{\prime}<a^{\prime \prime}, p_{a, a}=1_{X_{a}}$;

(A2) for each $a \in A$ and each normal cover $\mathcal{U}$ of $X_{a}$, there exists $a^{\prime}>a$ such that $d\left(p_{a, a_{1}} p_{a_{1}, a_{2}}, p_{a, a_{2}}\right)<\mathcal{U}$, whenever $a_{2}>a_{1}>a^{\prime}$

(A3) for each $a \in A$ and each normal cover $\mathcal{U}$ of $X_{a}$, there exists $a^{\prime}>a$ such that $\mathcal{U}_{a^{\prime \prime}}$ refines $p_{a, a^{\prime \prime}}^{-1} \mathcal{U}$ whenever $a^{\prime \prime}>a^{\prime}$.

For such a system, the (canonical) limit consists of all those points $x=\left(x_{a}\right) \in$ $\prod X_{a}$ with the property that for all $a \in A, \lim _{a^{\prime}>a} p_{a, a^{\prime}}\left(x_{a^{\prime}}\right)=x_{a}$.

Let us recall certain facts which are going to be used in our proof of 1.1.

2.1. Proposition. Every polyhedron of a full simplicial complex with the metric topology is an absolute retract (III.11.2 of $[\mathrm{Hu}]$ ).

2.2. Proposition. Every limit of an approximate system of compact Hausdorff spaces is an approximate resolution (4.2 of $[\mathrm{MW}]$ ).

It is worth explaining this last result. Suppose that $\mathbf{X}=\left\{X_{a}, \mathcal{U}_{a}, p_{a, a^{\prime}}, A\right\}$ is an approximate system of compacta $X_{a}$. Such a system always has a limit which is a compact Hausdorff space $X$ and such that $X \subset \prod\left\{X_{a} \mid a \in A\right\}$. This is referred to as the "canonical limit" in [MW]. We shall use only the canonical limit, and hence the approximate map $\mathbf{p}$ referred to in 4.2 of $[\mathrm{MW}]$ is just the collection of coordinate projections $p_{a}$ of $\prod\left\{X_{a} \mid a \in A\right\}$ restricted to $X$.

An approximate resolution enjoys two special properties, namely (R1) and (R2) of $[\mathrm{MW}]$, but we are concerned only with (R1) here. It goes this way. For every polyhedron $K$, open cover $\mathcal{V}$ of $K$, and map $F: X \rightarrow K$, there exists $a \in A$ such that for every $a^{\prime}>a$ there exists a map $h: X_{a^{\prime}} \rightarrow K$ such that $h p_{a^{\prime}}$ is $\mathcal{V}$-close to $F$. 
Proof of 1.1. Let $A$ be a closed subset of $X$, and let $f: A \rightarrow K$ be a map. Since $X$ is compact, $K$ is an absolute neighborhood extensor for $X$. Hence if we can find a map $g: A \rightarrow K$ which is homotopic to $f$ and such that $g$ extends to a map $G$ of $X$ to $K$, then our proof will be complete. By this and remarks above, we may as well assume that $K$ has the metric topology induced by its triangulation.

Let $\mathcal{U}$ be an open cover of $K$ such that any two maps of a space into $K$ which are st $\mathcal{U}$-close are homotopic. Now let $L$ designate the full complex on the vertices of $K$ (we shall not distinguish between a polyhedron and its triangulation), again with the metric topology induced by its triangulation. Of course this metric restricts to the original metric of $K$. According to $2.1, L$ is an absolute retract. Let $F: X \rightarrow L$ be a map which is an extension of the map $f$.

By 11.1 of $[\mathrm{Hu}], K$ is a neighborhood retract of $L$. Choose a retraction $r: W \rightarrow$ $K$ where $W$ is a neighborhood of $K$ in $L$. Now find an open cover $\mathcal{V}$ of $L$ whose restriction $\mathcal{U}_{0}$ on $K$ is a refinement of $\mathcal{U}$, and such that if $U \in \mathcal{V}$ and $U \cap K \neq \emptyset$, then $U \subset W$ and $r$ carries $U$ into an element of $\mathcal{U}$.

Apply 2.2 to get an index $a \in A$ and a map $h: X_{a} \rightarrow L$ such that $h p_{a}$ is $\mathcal{V}$-close to $F$. Consider the map $g: A \rightarrow K$ defined by the formula $x \rightarrow r\left(h\left(p_{a}(x)\right)\right)$. This is well-defined because $f(x)=F(x)$ and $h\left(p_{a}(x)\right)$ both lie in an element $U_{x}$ of $\mathcal{V}$ which thus must have nonempty intersection with $K$ and which therefore lies in $W$. One sees that the maps $g$ and $f$ are st $\mathcal{U}$-close, so they are homotopic.

On the other hand, the map $h_{1}: p_{a}(A) \rightarrow K$ given by $t \rightarrow r(h(t))$ is defined on the closed subset $p_{a}(A)$ of $X_{a}$. Since $X_{a} \tau K, h_{1}$ extends to a map $H_{1}: X_{a} \rightarrow K$. Now the map $G=H_{1} p_{a}: X \rightarrow K$ is an extension of $g$, so our proof is complete.

If $\mathbf{X}=\left\{X_{a}, p_{a, a^{\prime}}, A\right\}$ is an inverse system of compacta, then the projections $p_{a}$ : $X=\lim \mathbf{X} \rightarrow X_{a}$ form a resolution of $X$ (see Theorem 1 of I,6.1 of [MS]). Since resolutions satisfy the property (R1), we obtain the following as a corollary to our proof.

2.3. Corollary. Let $K$ be a polyhedron, let $\mathbf{X}=\left\{X_{a}, p_{a, a^{\prime}}, A\right\}$ be an inverse system of compacta such that for each $a \in A, X_{a} \tau K$, and let $X=\lim \mathbf{X}$. Then $X \tau K$. In particular if $\operatorname{dim} X_{a} \leq n$ (resp., $\operatorname{dim}_{G} X_{a} \leq n$ for a fixed abelian group $G$ ) for each $a \in A$, then $\operatorname{dim} X \leq n$ (resp., $\operatorname{dim}_{G} X \leq n$ ).

\section{REFERENCES}

[DD] A. Dranishnikov and J. Dydak, Extension theory of separable metrizable spaces with applications to dimension theory (preliminary version), preprint.

[DR] T. Dobrowolski and L. Rubin, The hyperspaces of infinite-dimensional compacta for covering and cohomological dimension are homeomorphic, Pacific J. of Math. 164 (1994), 15-39. MR 95a:54025

$[\mathrm{Hu}] \quad$ S. Hu, Theory of Retracts, Wayne State University Press, Detroit, 1965. MR 31:6202

[Ma] S. Mardešić, Approximate inverse systems and resolutions, Fundamenta Math. 142 (1993), 241-255. MR 94f:54025

[MR1] S. Mardešić and L. Rubin, Approximate inverse systems of compacta and covering dimension, Pacific J. of Math. 138 (1989), 129-144. MR 90f:54058

[MR2] S. Mardešić and L. Rubin, Cell-like mappings and nonmetrizable compacta of finite cohomological dimension, Trans. Amer. Math. Soc. 313 (1989), 53-79. MR 90a:54095

[MRU] S. Mardešić, L. Rubin, and N. Uglešić, A note on approximate systems of metric compacta, Topology and its Appls. 59 (1994), 189-194. MR 96a:54008

[MS] S. Mardešić and J. Segal, Shape Theory, North-Holland, Amsterdam, 1982. MR 84b:55020

[MW] S. Mardešić and T. Watanabe, Approximate resolutions of spaces and mappings, Glasnik Mat. 24 (1989), 583-633. MR 92b:54019 
[Na] K. Nagami, Dimension Theory, Academic Press, New York, 1970. MR 42:6799

[Wa] T. Watanabe, Numerical meshes and covering meshes of approximate inverse systems of compacta, Proc. Amer. Math. Soc. 123 (1995), 959-962. MR 95d:54007

[Wh] G. Whitehead, Elements of Homotopy Theory, Springer-Verlag, New York, 1978. MR 80b:55001

Department of Mathematics, University of Oklahoma, 601 Elm Ave., Rm. 423, NorMAN, OKLAHOMA 73019

E-mail address: LRUBIN@ou.edu 